\title{
Restoring Coronary Perfusion Pressure before Defibrillation after Chest Compression Interruptions
}

\author{
Timothy J. Mader*, Ryan A. Coute, Adam R. Kellogg, Joshua L. Harris, Scot A. Millay, \\ Leonard C. Jensen \\ Department of Emergency Medicine at Baystate Medical Center, Tufts University School of Medicine, \\ Springfield, USA \\ Email: ${ }^{*}$ timothy.mader@baystatehealth.org
}

Received 6 March 2014; revised 16 April 2014; accepted 20 May 2014

Copyright (C) 2014 by authors and Scientific Research Publishing Inc.

This work is licensed under the Creative Commons Attribution International License (CC BY). http://creativecommons.org/licenses/by/4.0/

\section{Open Access}

\section{Abstract}

Background: Sufficient coronary perfusion pressure (CPP) to provide myocardial reperfusion is required for defibrillation success after prolonged ventricular fibrillation (VF) cardiac arrest. Chest compression interruptions cause a precipitous drop in CPP. Objective: To quantify the extent to which CPP recovers to pre-pause levels following chest compression interruptions. Methods: This was a secondary analysis of data from two similar IACUC approved protocols. A total of 105 Yorkshire swine were included and VF was electrically induced. After 10 minutes of untreated VF in the first study $(n=52)$ and 12 minutes of untreated VF in the second $(n=53), C P R$ began and epinephrine was administered approximately 2 minutes prior to a planned 10 -second pause to record an artifact-free ECG waveform segment. Following this pause, CPR was resumed for 20seconds prior to defibrillation. CPP data were extracted from three time points: 2 minutes after epinephrine delivery (CPP1); following the chest compression pause (CPP2); and immediately before defibrillation (CPP3). Our primary outcome was defined as the ratio of CPP recovery (CPP3CPP2) to the drop in CPP (CPP1-CPP2). Results: Interrupting compressions resulted in a significant drop in CPP (29.8 mmHg [95\%CI: 26.2, 33.4] to $6.8 \mathrm{mmHg}$ [95\%CI: 5.4, 8.2]). Resuming CPR for restored $83 \%$ (95\%CI: 78\%, 86\%) of the CPP lost. Conclusion: This study demonstrates that $83 \%$ of the decline in CPP values during a planned 10-second interruption in CPR can be restored with a short period of precordial compressions prior to defibrillation.

\section{Keywords}

Cardiopulmonary Resuscitation, Coronary Perfusion Pressure, Chest Compression Interruptions

\footnotetext{
${ }^{*}$ Corresponding author.
}

How to cite this paper: Mader, T.J., Coute, R.A., Kellogg, A.R., Harris, J.L., Millay, S.A. and Jensen, L.C. (2014) Restoring Coronary Perfusion Pressure before Defibrillation after Chest Compression Interruptions. Open Journal of Emergency Medicine, 2, 29-35. http://dx.doi.org/10.4236/ojem.2014.22005 


\section{Introduction}

The importance of myocardial reperfusion, denoted by the achievement of a threshold coronary perfusion pressure (CPP), to defibrillation success beyond the electrical phase of ventricular fibrillation (VF) has been well established [1]-[15]. When chest compression interruptions occur during cardiopulmonary resuscitation (CPR), there is a precipitous and sustained loss of CPP [16] [17]. The aim of this study was to quantify the extent to which the decline in CPP values during an interruption in chest compressions for electrocardiogram (ECG) rhythm analysis can be restored with a short period of CPR prior to defibrillation. Our hypothesis was that a pause in chest compressions would lead to a precipitous drop in CPP values, but that delivering 30 chest compressions prior to defibrillation would restore a substantial proportion of the decay in CPP.

\section{Methods}

This was a secondary analysis of prospectively collected data from two similar IACUC approved protocols. Animal handling and all surgical procedures were in strict compliance with the NIH Guide for the Care and Use of Animals. Both studies were conducted in our USDA-certified laboratory. A total of 105 female Yorkshire swine (weighing 30 - $35 \mathrm{~kg}$ ) were included. From the first study, data from both the experimental (Group A [ $\mathrm{n}=$ 26]) and control (Group B [ $=26]$ ) groups were used because the groups were similarly treated [18]. From the second study, only the animals in the control group (Group C $[n=53]$ ) were included because the treatment $(n=$ 27) group received non-standard resuscitation medications that differed substantially from the other 3 groups. Both studies were included in the analysis to increase the generalizability and clinical application of our results.

The animal preparation methods for these experiments have been previously described in detail [18]. In both studies, animals were instrumented under general anesthesia after an intramuscular TKX [telazol ( $5 \mathrm{mg} / \mathrm{kg})$, ketamine $(2.5 \mathrm{mg} / \mathrm{kg})$, and xylazine $(2.5 \mathrm{mg} / \mathrm{kg})$ ] for sedation. The lead II electrocardiogram (ECG), as well as the arterial and venous pressures were monitored and recorded continually throughout the procedures. All data were acquired digitally at a sampling rate of 1000 points/second with a commercially available software package (Chart for Macintosh, AD Instruments, Colorado Springs, CO). Induction of VF was accomplished by delivering a three second, $60 \mathrm{~Hz}, 100 \mathrm{~mA}$ AC current externally across the thorax and mechanical ventilation was suspended.

After 10 minutes of untreated VF in the first study and 12 minutes of untreated VF in the second, resuscitation commenced with initiation of precordial chest compressions. Cardiopulmonary resuscitation was standardized in all groups by use of a pressure-driven mechanical resuscitation device (Thumper, Michigan Instruments, Grand Rapids, MI). Chest compressions were done at a rate of 100 compressions per minute, and a $50 \%$ duty cycle. Animals in Group B $(n=26)$ had continuous uninterrupted precordial chest compressions at 100/minute with no active ventilations or supplemental oxygen [18]. The compression to ventilation ratio in the other two groups (n = 79) was 30:2 with a $1.0 \mathrm{~F}_{\mathrm{i}} \mathrm{O}_{2}$. To minimize chest wall trauma, the compression depth was gradually adjusted over 20 seconds to generate peak aortic pressure spikes of $40-50 \mathrm{mmHg}$ (1.25 - 2 inches in depth). The duration of drug-free CPR varied by protocol (Table 1).

The animals in all three groups were given a premeasured standard $(0.01 \mathrm{mg} / \mathrm{kg})$ or high dose $(0.1 \mathrm{mg} / \mathrm{kg})$ of epinephrine approximately 2.0 minutes prior to a planned 10 -second resuscitation pause to record an artifact-free segment of the ECG tracing for offline VF quantitative waveform analysis [19] (Figure 1(a)). This pause was immediately followed by 30 compressions to further circulate the vasopressor and to restore CPP before the first rescue shock (RS) was delivered. If the RS failed, CPR resumed immediately, additional vasopressors were given, and the sequence repeated. Each rescue shock was delivered at a fixed dose of energy (120 J) with a proprietary Rectilinear Biphasic ${ }^{\mathrm{TM}}$ defibrillation waveform (E Series ${ }^{\mathrm{TM}}$, Zoll Medical Corp., Chelmsford, MA) through adult sized defibrillator pads (Adult Plus multifunction electrode pads, Philips Healthcare, Andover, MA).

The CPP for all subjects was computed as the arithmetic difference between the time-coincident aortic diastolic pressure and the right atrial pressure measured at the end of the relaxation phase of the duty cycle. Only the first RS attempt in each animal was examined. The values for analysis were extracted at three time points: 2 minutes after epinephrine delivery (CPP1); at the end of the 10-second pause (CPP2); and immediately before defibrillation (CPP3) (Figure 1(b)). A single investigator (TJM) was responsible for all CPP data extraction, recording, and analysis.

The primary outcome variable was defined as the ratio of CPP recovery with resuming chest compressions 
Table 1. Group characteristics presented as means (standard deviation).

\begin{tabular}{|c|c|c|c|c|}
\hline Group (n) & A (26) & B (26) & C (53) & All (105) \\
\hline Weight (kg) & $31.5(3.2)$ & $31.2(3.2)$ & $32.4(2.7)$ & $31.9(3.0)$ \\
\hline Anesthesia time (minutes) & $28.0(7.8)$ & $26.5(5.2)$ & $25.8(11.4)$ & $26.5(9.3)$ \\
\hline End tidal carbon dioxide (mmHg) & $40.1(1.6)$ & 40.7 (1.9) & $41.2(2.8)$ & $40.8(2.4)$ \\
\hline Temperature $\left({ }^{\circ} \mathrm{C}\right)$ & $37.8(0.9)$ & $37.7(0.8)$ & $37.6(0.9)$ & $37.7(0.8)$ \\
\hline Mean arterial pressure (mmHg) & $90.7(12.2)$ & $95.8(15.0)$ & $108.0(13.4)$ & $100.7(15.4)$ \\
\hline Heart rate (bpm) & $113.2(18.4)$ & $115.8(17.7)$ & $122.6(18.9)$ & $118.6(18.8)$ \\
\hline Glucose (mg/dL) & $89.6(34.5)$ & $101.0(44.9)$ & $92.7(23.2)$ & $94.1(33.4)$ \\
\hline Sodium (mEq/L) & $140.6(2.9)$ & $139.7(1.7)$ & $139.5(1.7)$ & $139.8(2.1)$ \\
\hline Potassium (mEq/L) & $4.0(0.3)$ & $4.0(0.3)$ & $4.0(0.4)$ & $4.0(0.3)$ \\
\hline Ionized calcium (mmol/L) & $1.37(0.07)$ & $1.37(0.07)$ & $1.38(0.06)$ & $1.37(0.06)$ \\
\hline Hematocrit (\%) & $26.1(2.8)$ & $26.7(4.2)$ & $27.7(2.4)$ & $27(3.1)$ \\
\hline $\mathrm{pH}$ (units) & $7.51(0.04)$ & $7.51(0.04)$ & $7.49(0.04)$ & $7.50(0.04)$ \\
\hline Partial pressure of $\mathrm{CO}_{2}(\mathrm{mmHg})$ & $36.9(3.0)$ & $37.3(3.1)$ & $40.1(3.3)$ & $38.6(3.4)$ \\
\hline Partial pressure of oxygen (mmHg) & $89.1(12.1)$ & $90.4(11.7)$ & $88.5(10.1)$ & $89.2(11.0)$ \\
\hline Untreated VF (minutes) & 10 & 10 & 12 & $10-12$ \\
\hline CPR (compressions/ventilations) & $30: 2$ & CCC & $30: 2$ & \\
\hline AoPr30 (mmHg) & $57.5(16.2)$ & $50.5(14.5)$ & $56.7(13.7)$ & $55.3(14.7)$ \\
\hline Drug-free CPR (minutes) & 6.5 & 0.5 & 0.5 & \\
\hline Epinephrine CPR (minutes) & 2.5 & 2.5 & 2.5 & 2.5 \\
\hline Epinephrine dose (mg/kg) & 0.1 & 0.1 & 0.01 & $0.01-0.1$ \\
\hline
\end{tabular}

CCC $=$ Continuous Chest Compressions; CPP = Coronary Perfusion Pressure; AoPr30 = Aortic pressure spike measures 30 seconds after initiation of precordial chest compressions.

after the 10-second pause (СРP3-СРP2) to the drop in CPP during the 10-second pause (CPP1-СРP2). Means and $95 \%$ confidence intervals were calculated for the main outcomes. All of the statistical calculations were performed using commercially available software (Stata/SE v. 11.0, College Station, TX). All data were assessed using descriptive statistics.

\section{Results}

Table 1 summarizes the characteristics of the cohort. The three groups were mathematically the same. The study findings are presented in Table 2. Overall, the pause in chest compressions lasted 12.8 seconds during which time an artifact-free segment of the ECG tracing was recorded for off-line analysis. Interrupting chest compressions to analyze the ECG VF rhythm caused a significant drop in CPP from $29.8 \mathrm{mmHg}$ to $6.8 \mathrm{mmHg}$. Resuming CPR for 20 seconds prior to RS delivery restored 83\% (95\%CI: 78\%, 86\%) of the CPP lost during the CPR interruption. This is presented graphically in the box plot (Figure 2).

\section{Discussion}

The generation of CPP adequate to provide myocardial reperfusion (typically quoted as $15-25 \mathrm{mmHg}$ ) after prolonged VF cardiac arrest is critical to defibrillation success [1]-[15]. The delivery of high quality continuous chest compressions during CPR provides cerebral and myocardial blood flow and increases the probability of survival after sudden unexpected collapse [20] [21]. Conversely, poor quality chest compressions with excessive 


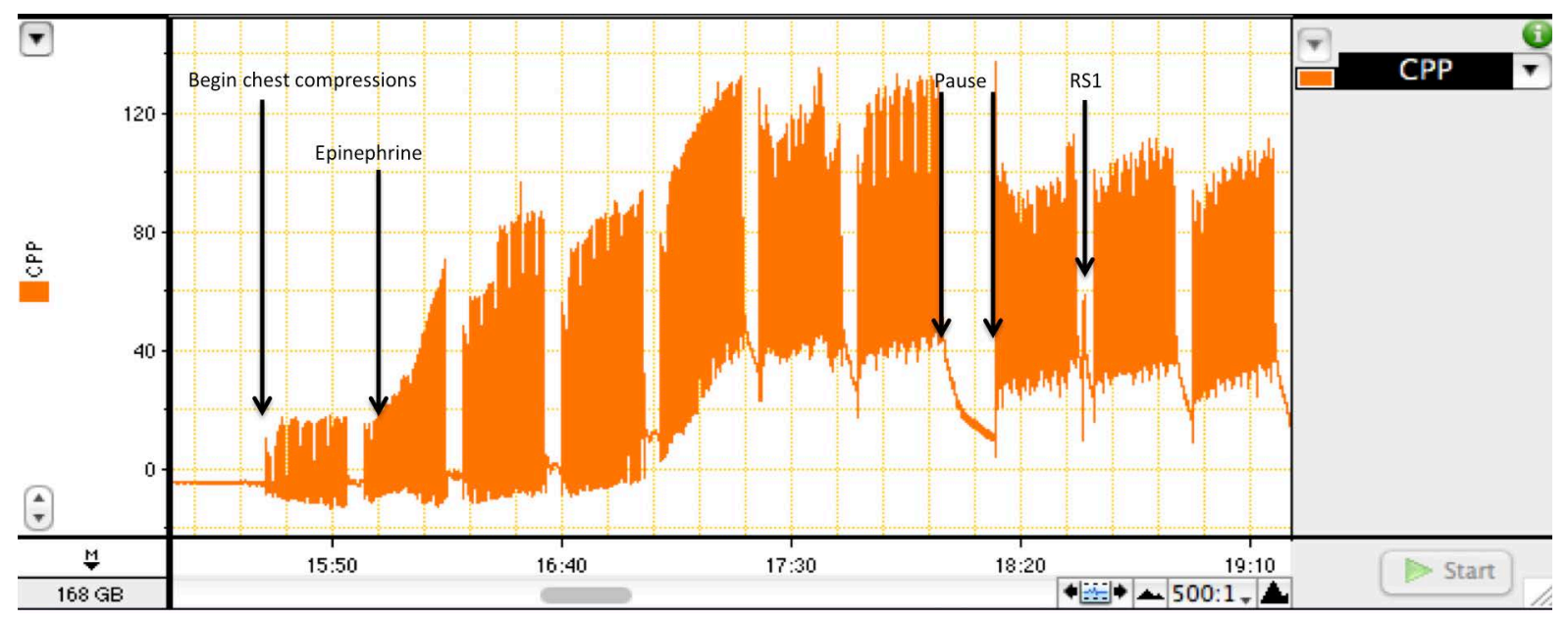

(a)

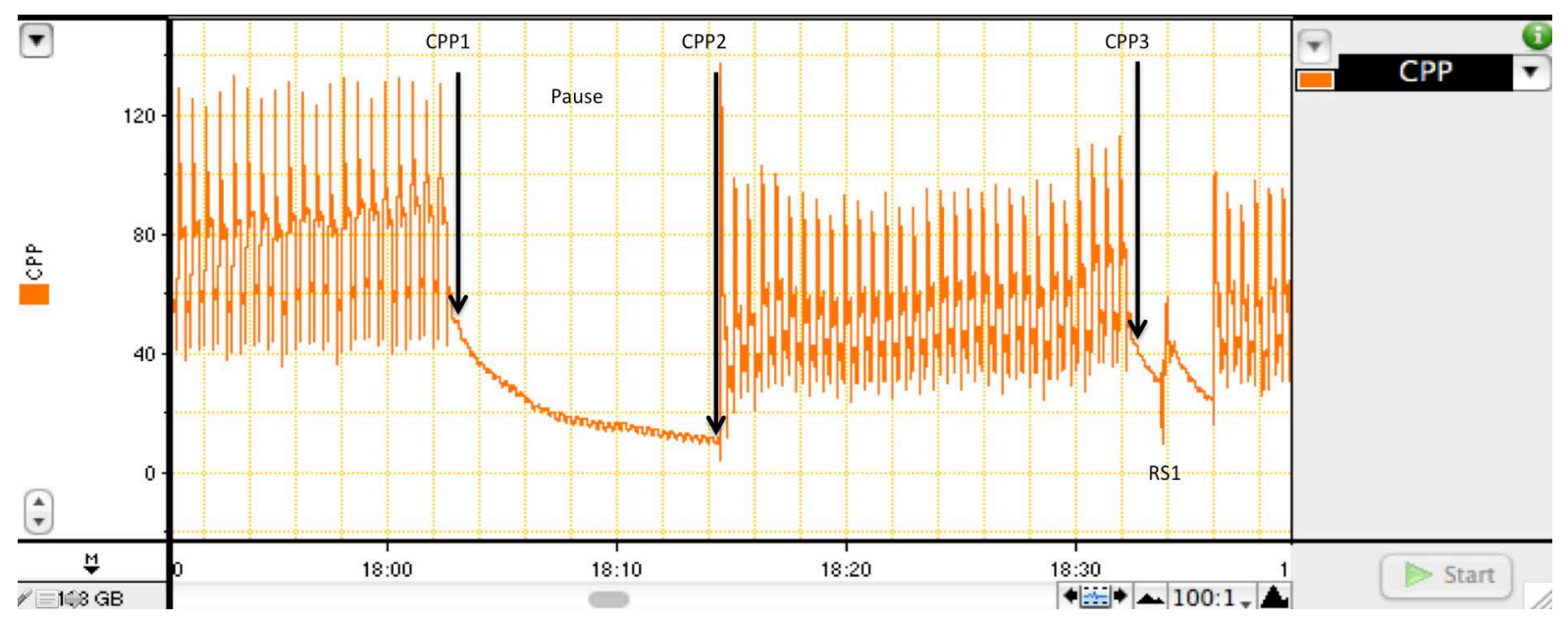

(b)

Figure 1. A segment of the CPP tracing from a representative animal in Group C demonstrating the resuscitation sequence and the selection of the CPP measurements. (a) After 12 minutes of untreated VF, resuscitation commenced with initiation of precordial chest compressions. In this experiment, 30 seconds of basic CPR simulated the time required to establish intraosseous access for vasopressor delivery [18]. The epinephrine was actually injected through a central venous line and flushed with a large volume of normal saline. Following 7 sets of precordial chest compressions and ventilations at a ratio of 30:2, a planned 10-second CPR pause allowed for recording of an artifact-free segment of the ECG channel. This pause was immediately followed by another set of precordial chest compressions to restore CPP before the first RS was delivered. The time from CPP3 to $\mathrm{RS}_{1}$ is $\sim 1.5 \mathrm{sec}$; (b) An enlargement of the tracing surrounding the pause illustrates the 3 points at which the CPP measurements were extracted in relation to the timing of the first RS.

Table 2. Group and overall outcomes presented as means, $50^{\text {th }}$ percentile, and proportions (95\% confidence intervals).

\begin{tabular}{ccccc}
\hline Group (n) & A (26) & B (26) & C (53) & All (105) \\
\hline Pause (seconds) & $14.7(13.7,15.6)$ & $10.2(9.8,10.6)$ & $13.2(12.6,13.8)$ & $12.8(12.3,13.3)$ \\
CPP1 (mmHg) & $26.7(18.9,34.5)$ & $36.9(28.8,45.0)$ & $27.9(23.2,32.5)$ & $29.8(26.2,33.4)$ \\
CPP2 (mmHg) & $6.6(3.5,9.8)$ & $9.6(6.5,12.6)$ & $5.5(3.8,7.2)$ & $6.8(5.4,8.2)$ \\
CPP3 (mmHg) & $21.5(14.7,28.3)$ & $33.2(26.6,40.0)$ & $25.0(20.5,29.4)$ & $26.2(22.9,29.4)$ \\
Ratio & $0.79(0.58,0.86)$ & $0.83(0.73,0.87)$ & $0.85(0.76,0.92)$ & $0.83(0.78,0.86)$ \\
\hline
\end{tabular}

$\mathrm{CPP}=$ Coronary Perfusion Pressure; Ratio $=(\mathrm{CPP} 3-\mathrm{CPP} 2) /(\mathrm{CPP} 1-\mathrm{CPP} 2)$. 


\section{Mean CPP values (95\% $\mathrm{Cl}$ error bars)}

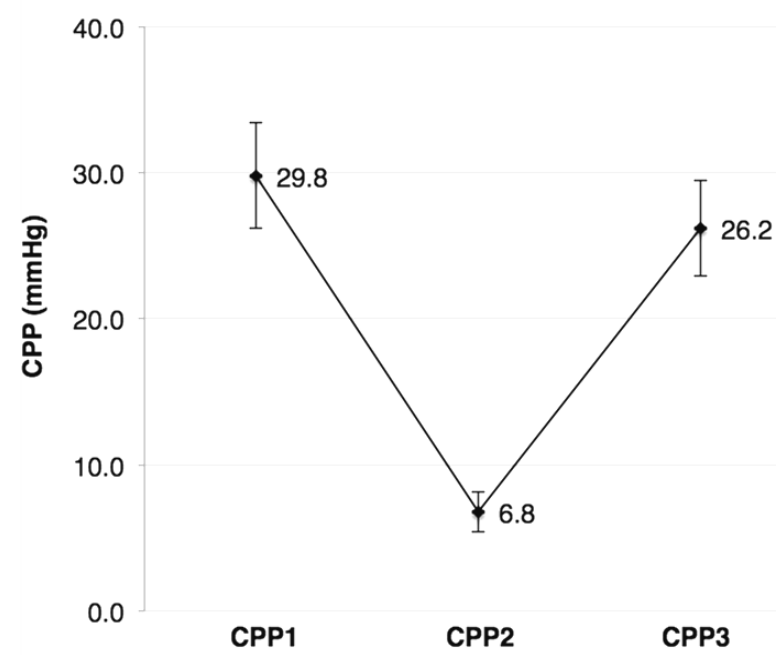

Figure 2. The graphical depiction of CPP values at each of the time point. At the beginning of the pause, the mean CPP (CPP1) was $29.8 \mathrm{mmHg}$ (95\%CI: 26.2, 33.4). After recording the artifact-free segment of the ECG signal, the mean CPP (CPP2) was $6.8 \mathrm{mmHg}$ (95\%CI: 5.4, 8.2) - a 77\% loss of the pre-pause measurement. Resuming chest compressions for 20 seconds prior to RS delivery restored $83 \%$ of the CPP lost during the 10 -second interruption in chest compressions (88\% of the pre-pause CPP value), resulting in a mean of 26.2 mmHg (95\%CI: 22.9, 29.4)—a value more favorable to RS success.

interruptions lowers the chances of terminating VF and achieving return of spontaneous circulation (ROSC), which hinders survival [22]-[24].

Some chest compression interruptions (i.e., to perform procedures, for automatic or visual ECG rhythm analysis, and to perform defibrillation) may be unavoidable. We have previously examined the effect of a brief preshock pause (PSP) in precordial chest compressions on CPP decay in 120 swine after prolonged VF and noted a precipitous and sustained loss of CPP [17]. Overall, the mean CPP in that study decreased from 23.4 to 7.6 mmHg (a 68\% drop) following a median PSP of just 4.1 seconds. In the current study, we again noted a substantial decline in mean CPP from a pre-pause value of $29.8 \mathrm{mmHg}$ to a low of $6.8 \mathrm{mmHg}$ (a drop of 77\%) after 10 seconds of interrupting CPR, but then go on to describe the extent to which CPP can be restored with a set of precordial chest compressions prior to defibrillation.

To our knowledge, this is the first study to describe the effect of resuming precordial chest compressions after a brief pause in CPR to restore CPP prior to defibrillation. The importance of this observational study to human VF cardiac arrest resuscitation and whether or not this intervention might translate into greater RS success, higher rates of ROSC, and improved survival remains to be determined.

In this regard, research published by Sato et al. [16], may provide some insights. Using a murine VF cardiac arrest model, their team randomized animals to a PSP of 0, 10, 20, 30, or 40 seconds after four minutes of untreated VF. Those with no delay between CPR and defibrillation had a mean CPP of $26 \pm 2 \mathrm{mmHg}$ and 5/5 achieved ROSC while $4 / 5$ survived to 24 hours. In the group that had a 10-second pause between CPR and defibrillation, the mean CPP was $6 \pm 3 \mathrm{mmHg}$ (coincidently, a 77\% drop) at the time of defibrillation and 3/5 achieved ROSC but only 2/5 survived to 24 hours. Longer delays between CPR and defibrillation resulted in similarly low CPP values ( $4 \pm 2,4 \pm 4,4 \pm 4 \mathrm{mmHg}$, respectively) at the time of defibrillation but statistically significant lower rates of ROSC (3/5, $1 / 5,0 / 5$, respectively) and 24-hour survival (0/5, 0/5, 0/5, respectively). These data suggest that the CPP at the time of defibrillation rather than a threshold CPP value exceeded [10], maximum CPP achieved [15], or cumulative CPP dose delivered [25] prior to defibrillation is important to RS success. 


\section{Limitations}

There are several important limitations to this study. First, this was a retrospective analysis of pooled data from studies using a swine model of prolonged VF arrest using young healthy animals. How well our findings apply to human cardiac arrest is debatable given the co-morbidities of the typical cardiac arrest patient, which have profound effects on the cardiovascular system. Second, VF in our animal model of cardiac arrest was electrically induced while ischemic VF may be more clinically applicable. Finally, all data points were extracted by a single investigator (TJM), however to minimize bias, pressure measures were taken from a consistent predetermined point on each tracing leaving little room for discretion.

\section{Conclusion}

This observational study using experimental swine data demonstrates that $83 \%$ of the decline in CPP values during a planned 10-second interruption in CPR can be restored with a short period of precordial chest compressions prior to defibrillation. The importance of these findings to human VF cardiac arrest resuscitation remains to be determined. Pending further investigation, it appears advisable to provide a brief period of chest compressions prior to defibrillation to restore CPP after an intentional or inadvertent pause in CPR of more than a few seconds duration.

\section{Funding Statement}

Project grants through the Baystate Health Incubator Fund Grant Program supported the studies from which these data were extracted.

\section{Contributors}

TJM designed the study, supervised data collection, performed the data analysis, and wrote the manuscript. RAC assisted in writing the manuscript and performed data collection. ARK, SAM, and LCJ were all involved in data collection. TJM is responsible for this work as guarantor.

\section{Conflict of Interest}

None of the authors have a conflict of interest to report.

\section{References}

[1] Redding, J.S. and Pearson, J.W. (1963) Evaluation of Drugs for Cardiac Resuscitation. Anesthesiology, 24, $203-207$. http://dx.doi.org/10.1097/00000542-196303000-00008

[2] Babbs, C.F. (1980) New versus Old Theories of Blood Flow during CPR. Critical Care Medicine, 8, 191-195. http://dx.doi.org/10.1097/00003246-198003000-00026

[3] Ditchey, R.V., Winkler, J.V. and Rhodes, C.A. (1982) Relative Lack of Coronary Blood Flow during Closed-Chest Resuscitation in Dogs. Circulation, 66, 297-302. http://dx.doi.org/10.1161/01.CIR.66.2.297

[4] Sanders, A.B., Ewy, G.A., Alferness, C.A., et al. (1982) Failure of One Method of Simultaneous Chest Compression, Ventilation, and Abdominal Binding during CPR. Critical Care Medicine, 10, 509-513. http://dx.doi.org/10.1097/00003246-198208000-00005

[5] Michael, J.R., Guerci, A.D., Koehler, R.C., et al. (1984) Mechanisms by Which Epinephrine Augments Cerebral and Myocardial Perfusion during Cardiopulmonary Resuscitation in Dogs. Circulation, 69, 822-835. http://dx.doi.org/10.1161/01.CIR.69.4.822

[6] Niemann, J.T., Rosborough, J.P., Niskanen, R.A., et al. (1985) Mechanical “Cough” Cardiopulmonary Resuscitation during Cardiac Arrest in Dogs. American Journal of Cardiology, 55, 199-204. http://dx.doi.org/10.1016/0002-9149(85)90328-5

[7] Niemann, J.T., Criley, J.M., Rosborough, J.P., et al. (1985) Predictive Indices of Successful Cardiac Resuscitation after Prolonged Arrest and Experimental Cardiopulmonary Resuscitation. Annals of Emergency Medicine, 14, 521-528. http://dx.doi.org/10.1016/S0196-0644(85)80774-5

[8] Sanders, A.B., Kern, K.B., Atlas, M., et al. (1985) Importance of the Duration of Inadequate Coronary Perfusion Pressure on Resuscitation from Cardiac Arrest. Journal of the American College of Cardiology, 6, 113-118. http://dx.doi.org/10.1016/S0735-1097(85)80261-8 
[9] Kern, K.B., Ewy, G.A., Voorhees, W.D., et al. (1988) Myocardial Perfusion Pressure: A Predictor of 24-Hour Survival during Prolonged Cardiac Arrest in Dogs. Resuscitation, 16, 241-250. http://dx.doi.org/10.1016/0300-9572(88)90111-6

[10] Paradis, N.A., Martin, G.B., Rivers, E.P., et al. (1990) Coronary Perfusion Pressure and the Return of Spontaneous Circulation in Human Cardiopulmonary Resuscitation. JAMA, 263, 1106-1113. http://dx.doi.org/10.1001/jama.1990.03440080084029

[11] Cobb, L.A., Fahrenbruch, C.E., Walsh, T.R., et al. (1999) Influence of Cardiopulmonary Resuscitation Prior to Defibrillation in Patients with Out-of-Hospital Ventricular Fibrillation. JAMA, 281, 1182-1188. http://dx.doi.org/10.1001/jama.281.13.1182

[12] Weisfeldt, M.L. and Becker, L.B. (2002) Resuscitation after Cardiac Arrest: A 3-Phase Time-Sensitive Model. JAMA, 288, 3035-3038. http://dx.doi.org/10.1001/jama.288.23.3035

[13] Wik, L., Hansen, T.B., Fylling, F., et al. (2003) Delaying Defibrillation to Give Basic Cardiopulmonary Resuscitation to Patients with Out-of-Hospital Ventricular Fibrillation: A Randomized Trial. JAMA, 289, 1389-1395. http://dx.doi.org/10.1001/jama.289.11.1389

[14] Berg, R.A., Hilwig, R.W., Ewy, G.A., et al. (2004) Precountershock Cardiopulmonary Resuscitation Improves Initial Response to Defibrillation from Prolonged Ventricular Fibrillation: A Randomized, Controlled Swine Study. Critical Care Medicine, 32, 1352-1357. http://dx.doi.org/10.1097/01.CCM.0000127780.01362.E5

[15] Reynolds, J.C., Salcido, D.D. and Menegazzi, J.J. (2010) Coronary Perfusion Pressure and Return of Spontaneous Circulation after Prolonged Cardiac Arrest. Prehospital Emergency Care, 14, 78-84. http://dx.doi.org/10.3109/10903120903349796

[16] Sato, Y., Weil, M.H., Sun, S., et al. (1997) Adverse Effects of Interrupting Precordial Compression during Cardiopulmonary Resuscitation. Critical Care Medicine, 25, 733-736. http://dx.doi.org/10.1097/00003246-199705000-00005

[17] Mader, T.J., Paquette, A.T., Salcido, D.D., et al. (2009) The Effect of the Preshock Pause on Coronary Perfusion Pressure Decay and Rescue Shock Outcome in Porcine Ventricular Fibrillation. Prehospital Emergency Care, 13, 487-494. http://dx.doi.org/10.1080/10903120903144916

[18] Mader, T.J., Kellogg, A.R., Walterscheid, J.K., et al. (2010) A Randomized Comparison of Cardiocerebral and Cardiopulmonary Resuscitation Using a Swine Model of Prolonged Ventricular Fibrillation. Resuscitation, 81, 596-602. http://dx.doi.org/10.1016/j.resuscitation.2010.01.013

[19] Salcido, D.D., Kim, Y.M., Sherman, L.D., et al. (2012) Quantitative Waveform Measures of the Electrocardiogram as Continuous Physiologic Feedback during Resuscitation with Cardiopulmonary Bypass. Resuscitation, 83, 505-510. http://dx.doi.org/10.1016/j.resuscitation.2011.09.018

[20] (2005) American Heart Association Guidelines for Cardiopulmonary Resuscitation and Emergency Cardiovascular Care. Circulation, 112, IV19-IV34.

[21] Bossaert, L. and Van Hoeyweghen, R. (1989) Bystander Cardiopulmonary Resuscitation (CPR) in Out-of-Hospital Cardiac Arrest. The Cerebral Resuscitation Study Group. Resuscitation, 17, S55-S69.

[22] Abella, B.S., Alvarado, J.P., Myklebust, H., et al. (2005) Quality of Cardiopulmonary Resuscitation during In-Hospital Cardiac Arrest. JAMA, 293, 305-310. http://dx.doi.org/10.1001/jama.293.3.305

[23] Wik, L., Kramer-Johansen, J., Myklebust, H., et al. (2005) Quality of Cardiopulmonary Resuscitation during Out-ofHospital Cardiac Arrest. JAMA, 293, 299-304. http://dx.doi.org/10.1001/jama.293.3.299

[24] Edelson, D.P., Abella, B.S., Kramer-Johansen, J., et al. (2006) Effects of Compression Depth and Pre-Shock Pauses Predict Defibrillation Failure during Cardiac Arrest. Resuscitation, 71, 137-145. http://dx.doi.org/10.1016/j.resuscitation.2006.04.008

[25] Reynolds, J.C., Salcido, D.D. and Menegazzi, J.J. (2012) Conceptual Models of Coronary Perfusion Pressure and Their Relationship to Defibrillation Success in a Porcine Model of Prolonged Out-of-Hospital Cardiac Arrest. Resuscitation, 83, 900-906. http://dx.doi.org/10.1016/j.resuscitation.2012.01.007 